Int. J. Morphol., 36(3):841-847, 2018.

\title{
Dientes Fósiles de Carcharocles megalodon: La Colección del Museo Universitario de Chieti (Italia). Parte II: Análisis Paleopatológico
}

\author{
Fossil Teeth of Carcharocles megalodon: The Collection of the \\ University Museum of Chieti (Italy). Part II: Paleopathological Analysis
}

Ruggero D’Anastasio'; Sandra López-Lázaro² \& Joan Viciano ${ }^{1}$

D’ANASTASIO, R.; LÓPEZ-LÁZARO, S. \& VICIANO, J. Dientes fósiles de Carcharocles megalodon: La colección del museo Universitario de Chieti (Italia). Parte II: Análisis paleopatológico. Int. J. Morphol., 36(3):841-847, 2018.

RESUMEN. La paleopatología es la ciencia que estudia el origen y la evolución de las enfermedades del pasado. El interés por esta disciplina deriva del hecho de que la frecuencia y la distribución de las patologías que han afectado a las especies extintas se correlacionan con su ecología y etología. Esta suposición también es válida en el caso de Carcharocles megalodon, el tiburón más grande jamás existido, situado en lo alto de la cadena alimenticia y que vivió en los mares templados desde el Mioceno medio hasta el Plioceno (15,9-2,6 Ma). En el presente artículo presentamos las alteraciones patológicas de los dientes de Carcharocles megalodon que se conservan en el Museo Universitario de Chieti (Italia). La colección se compone de nueve dientes de la arcada superior, todos ellos con presencia de alteraciones patológicas correspondientes a defectos del desarrollo y traumas de la corona. Un diente en particular (espécimen \#165), presenta una aposición anómala del esmalte sobre la superficie labial, que corresponde a una condición no descrita en la literatura. La colección del Museo Universitario de Chieti enriquece el registro paleopatológico relativamente pobre existente, contribuyendo a completar el conocimiento sobre la paleobiología de Carcharocles megalodon.

PALABRAS CLAVE: Megalodonte; Diente fósil; Lamniformes; Paleopatología; Anomalía congénita; Trauma.

\section{INTRODUCCIÓN}

La paleopatología (que deriva etimológicamente de los términos griegos $\pi \alpha \lambda \alpha$ lós —paleo, "antiguo, viejo"-,

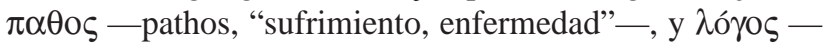
logos, "tratado, estudio, indagación"-) es definida como la ciencia que estudia las enfermedades del pasado y su evolución en el tiempo. Los materiales objeto de estudio de esta disciplina consisten principalmente en (i) fuentes indirectas, representadas por textos antiguos o restos de la cultura material y, por tanto, estrictamente limitadas a la esfera de la paleopatología humana, y (ii) fuentes directas, consistentes principalmente en restos biológicos antiguos de origen humano (huesos, dientes, momias, tejidos blandos calcificados), así como de restos no humanos (huesos fosilizados de animales y vegetales, coprolitos, parásitos y vectores de enfermedades infecciosas conservados en ámbar) (p. ej.: D'Anastasio et al., 2009, 2013; de Buffrénil et al., 2010; De Luca et al., 2013; Bedosti et al., 2015; Viciano et al., 2015a,c;
2017; Cilli \& D'Anastasio, 2017). Respecto al estudio de las enfermedades en la Prehistoria, los huesos y los dientes fósiles constituyen las únicas fuentes directas con las que poder extrapolar información sobre la naturaleza y la epidemiología de las enfermedades antiguas. Los dientes, en particular, conservan mucha información sobre el perfil biológico (Viciano et al., 2015b) y el estado de salud/enfermedad del ser humano (Viciano et al., 2012), así como de otros animales del pasado próximo y remoto (p. ej.: Capasso \& D'Anastasio, 2001; D'Anastasio et al., 2002; Böhmer \& Rössner, 2018). En el caso de los condrictios (Chondrichthyes), también conocidos como peces cartilaginosos, entre los que recordamos especies vivas como tiburones, rayas y mantarrayas, y especies extintas como el tiburón megalodonte, los dientes constituyen casi exclusivamente el registro fósil conservado de esta clase de vertebrados acuáticos, precisamente a causa de la naturaleza cartilaginosa de su esqueleto.

${ }^{1}$ Unidad Operativa de Antropología, Departamento de Medicina y Ciencias del Envejecimiento, Universidad "G. d'Annunzio "de Chieti-Pescara, Italia. ${ }^{2}$ Laboratorio de Odontología Forense, Centro de Investigación en Odontología Legal y Forense -CIO-, Facultad de Odontología, Universidad de La Frontera, Temuco, Chile. 
Las patologías actuales más frecuentes entre los condrictios parecen ser las alteraciones morfológicas de naturaleza congénita del esqueleto cartilaginoso y de las aletas (Dawson, 1964; Honma \& Sugihara, 1971; Heupel et al., 1999; Mancini et al., 2006; Ramírez Hernández et al., 2011). Por ejemplo, Schlumberger \& Lucke (1948) describieron un fibrosarcoma en el primer rayo de una aleta de un ejemplar de raya Raja macrorhyncha, y un condroma en una vértebra lumbar de un galludo espinilla (Squalus mitsukurii). Harshbarger (1980) diagnóstico una enfermedad endocrina conocida como bocio (sobrecrecimiento de la glándula tiroides pero con una hipofunción de la misma) en una pastinaca del género Dasyatis. Hoenig \& Walsh (1983) publicaron el caso de un tiburón trozo (Carcharhinus plumbeus) con centros vertebrales, costillas y arcos neurales fusionados, y con una deposición periférica de material calcificado sin modificación de la estructura básica de los centros vertebrales, probablemente de etiología infecciosa.

No obstante, los casos paleopatológicos relacionados con los condrictios y publicados en la literatura son limitados y extremadamente raros cuando se hace referencia a las alteraciones dentales. Hubbell (1996) dice haber recolectado y analizado más de 13.000 dientes fósiles del gran tiburón blanco Carcharodon carcharias, de los que solo el $0,25 \%$ presentaba alteraciones morfológicas, además de presentar una colección personal de 559 dientes de Carcharocles megalodon y Carcharodon auriculatus con deformidades de varia naturaleza. Sin embargo, su publicación no contiene la descripción de los dientes patológicos y la discusión de su posible significado biológico y ecológico. Renz (2002) presenta una serie de imágenes relacionadas con 14 dientes fósiles de C. megalodon de colecciones privadas, con alteraciones patológicas que incluyen ondulaciones del margen cortante, deformaciones congénitas de la corona y de la raíz, y aberraciones de la distribución y del número de las aserraduras en los márgenes mesial y distal.

La reconstrucción de la patocenosis de Carcharocles megalodon (Clase: Chondrichthyes; Orden: Lamniformes), es decir, del conjunto de enfermedades que afectaron a esta especie durante el transcurso de su historia evolutiva (Capasso, 2002), por el momento solo se basa en el diagnóstico de alteraciones patológicas encontradas en dientes fósiles (Leriche, 1926; Applegate \& Espinosa-Arrubarrena, 1996; Hubbell; Reolid \& Molina, 2015). Principalmente esto es debido a que los sedimentos fosilíferos solo han aportado dientes fósiles y algunos centros vertebrales de este gran depredador que pobló los mares templados de todo el mundo entre el Mioceno medio y el Plioceno (15,92,6 millones de años) (Leriche; Uyeno et al., 1989; Purdy et al., 2001; Pimiento \& Balk, 2015; Reolid \& Molina).
El Museo Universitario de la Universidad "G. d'Annunzio" de Chieti-Pescara (Italia), dedicado al conocimiento y divulgación de la historia de las ciencias biomédicas, posee una colección de dientes de C. megalodon compuesta por nueve especímenes. El artículo previamente publicado por Viciano et al. (2018) presenta su clasificación, sus características morfológicas y métricas, así como la estimación del tamaño y la masa corporal de los ejemplares a los que pertenecerían estos dientes. Esta segunda parte tiene como objetivo completar la información aportada previamente ilustrando las patologías que presentan tales dientes. Considerando que la manifestación de una patología, además de las características genéticas del huésped y del agente (en el caso de que se trate de enfermedades infecciosas), depende también de las características del ecosistema (Capasso; Rothschild \& Martin, 1993), la reconstrucción de la patocenosis de C. megalodon podría proporcionar información útil para comprender su paleobiología, su capacidad de adaptación y su papel en el ecosistema.

\section{MATERIAL Y MÉTODO}

Los nueve dientes de C. megalodon incluidos en la colección odontológica del Museo Universitario de Chieti proceden del yacimiento paleontológico de Lee Creek Mine (Carolina del Norte, EE.UU.) (Purdy et al.). Los especímenes mencionados en este artículo están inventariados con los códigos \#118, \#119, \#120, \#165, \#180, \#188, \#190, \#191 y \#192.

La nomenclatura adoptada es la propuesta por Applegate (1965), posteriormente modificada por Applegate \& Espinosa-Arrubarrena.

El diagnóstico de las alteraciones patológicas se basa en el análisis macroscópico y microscópico realizado con ayuda de un estereomicroscopio Leica Wild M8 con una ampliación de $\times 5$ a $\times 100$, bajo un sistema de iluminación Volpi Intralux 5000, y mediante comparación con los casos paleopatológicos citados en la literatura (Hubbell; Renz).

\section{RESULTADOS}

Los nueve dientes de Carcharocles megalodon presentan alteraciones patológicas de diversa naturaleza.

Espécimen \#118 (AIII; tercer diente anterior superior). El diente está íntegro, con la única ausencia del ápice de la corona a causa de una rotura tafonómica. El margen 
mesial de la corona, en proximidad del cuello dental, presenta una conformación ondulada (Fig. 1).

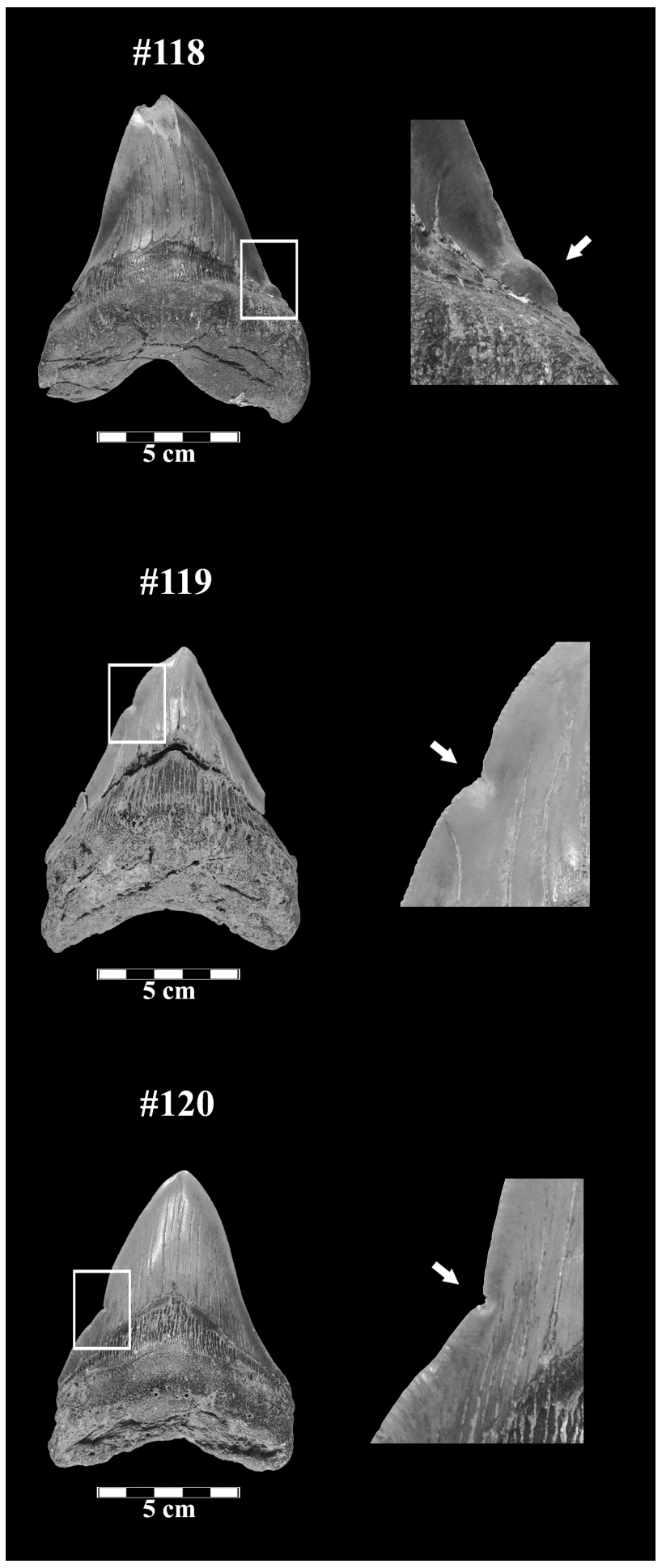

Fig. 1. Vista lingual y en detalle de los especímenes \#118, \#119 y \#120 de Carcharocles megalodon. El espécimen \#118 presenta una conformación ondulada del margen mesial de la corona. Los especímenes \#119 y \#120 presentan una pequeña hendidura en el margen mesial.
Espécimen \#119 (UL2(?); probablemente un segundo lateral superior). El diente está íntegro, con la única ausencia de la parte más basal del margen distal de la corona. Además, falta parte del revestimiento de esmalte en la superficie lingual en proximidad del cuello dental, a causa de una fractura de origen tafonómico. Ambos márgenes mesial y distal presentan una morfología normal, a excepción de una única alteración patológica que consiste en una pequeña hendidura en el margen mesial, en proximidad del ápice del diente (Fig. 1).

Espécimen \#120 (AI; primer diente anterior superior). El diente está íntegro y los márgenes mesial y distal son normales en morfología. Al igual que en el espécimen \#119, la única alteración patológica observada consiste en una pequeña hendidura en el margen mesial, pero a diferencia de éste, la hendidura se encuentra en proximidad del cuello dental (Fig. 1).

Espécimen \#165 (Int(?); probablemente un diente intermedio superior). El diente está íntegro y los márgenes mesial y distal son normales en morfología. La superficie labial de la corona presenta una aposición anómala de esmalte, que emerge de la superficie. La alteración tiene una forma elipsoidal, con una superficie lisa, y el borde mesial elevado. Se localiza justo debajo del ápice de la corona, a lo largo de la línea media del diente. La alteración tiene una longitud máxima de 2,2 cm y una anchura de $0,8 \mathrm{~cm}$ (Fig. 2).

Espécimen \#180 (UL6/7; probablemente un sexto o un séptimo diente lateral superior). Falta por completo la mitad mesial de la corona a causa, probablemente, de una rotura de origen tafonómico. El margen distal de la corona presenta una proyección puntiforme localizada a medio camino entre el ápice del diente y su cuello anatómico, cambiando marcadamente el perfil del diente. No se observan ondulaciones de los márgenes ni alteraciones de la superficie del esmalte (Fig. 2).

Espécimen \#188 (AI; primer diente anterior superior). A causa, probablemente, de una fractura de origen tafonómico, falta parte del revestimiento de esmalte en la superficie lingual. De acuerdo con la anatomía general, la superficie labial de la corona de los dientes superiores de C. megalodon generalmente tiene un perfil cóncavo o plano (Applegate \& Espinosa-Arrubarrena). El espécimen \#188 tiene una superficie labial ondulada (muy evidente cuando el diente se observa de perfil), teniendo los márgenes mesial y distal una morfología normal (Fig. 2).

Espécimen \#190 (AI; primer diente anterior superior). Se observan alteraciones de origen tafonómico ya sea en la corona como en la raíz. En particular, falta parte del revestimiento de esmalte tanto sobre la superficie labial como sobre la lingual, así como están ausentes el lóbulo radicular y parte 


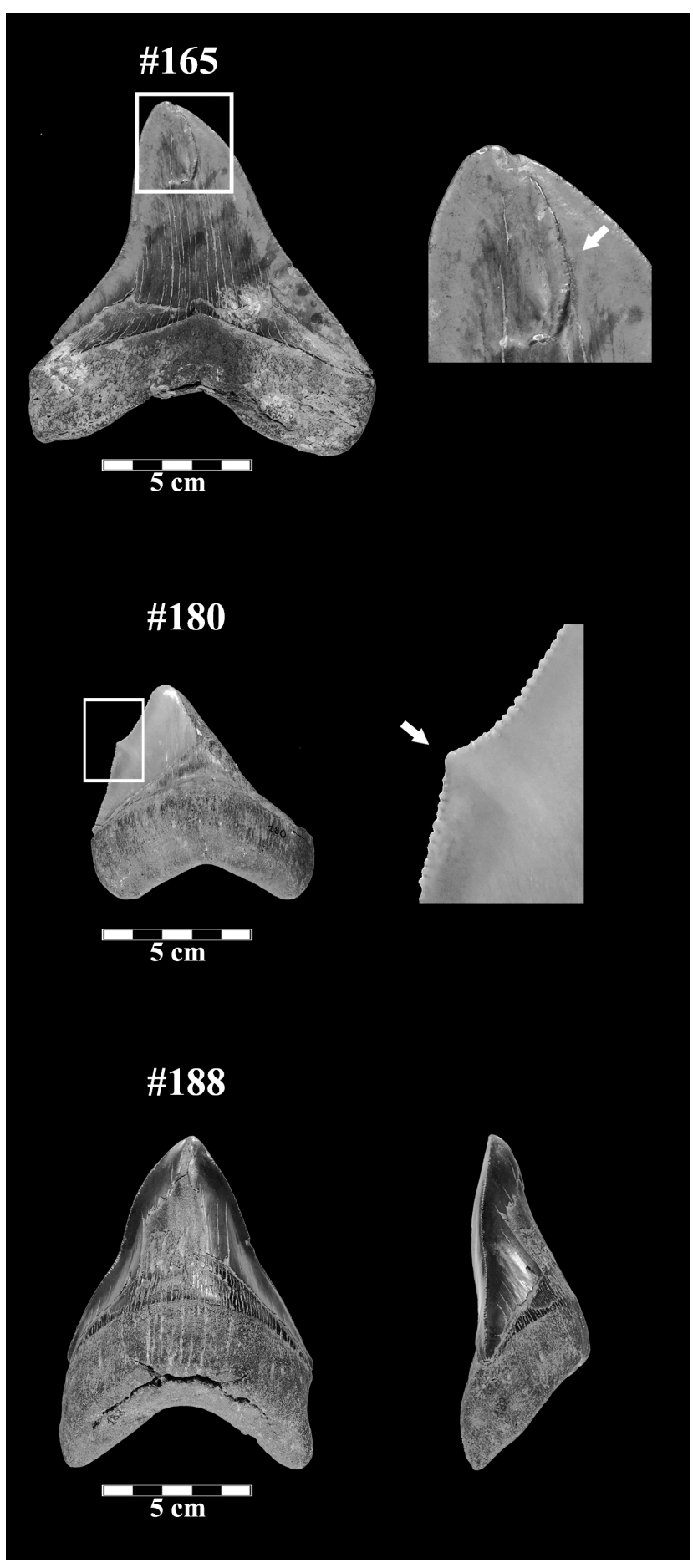

Fig. 2. Vista labial (espécimen \#165), lingual (especímenes \#180 y \#188) y en detalle de los dientes de Carcharocles megalodon. El espécimen \#165 presenta una aposición anómala de esmalte, que emerge de la superficie. El espécimen \#180 presenta una proyección puntiforme localizada en el margen distal, a medio camino entre el ápice del diente y su cuello anatómico, cambiando marcadamente el perfil del diente. El espécimen \#188, visto de perfil, presenta una superficie labial con un contorno ondulado.
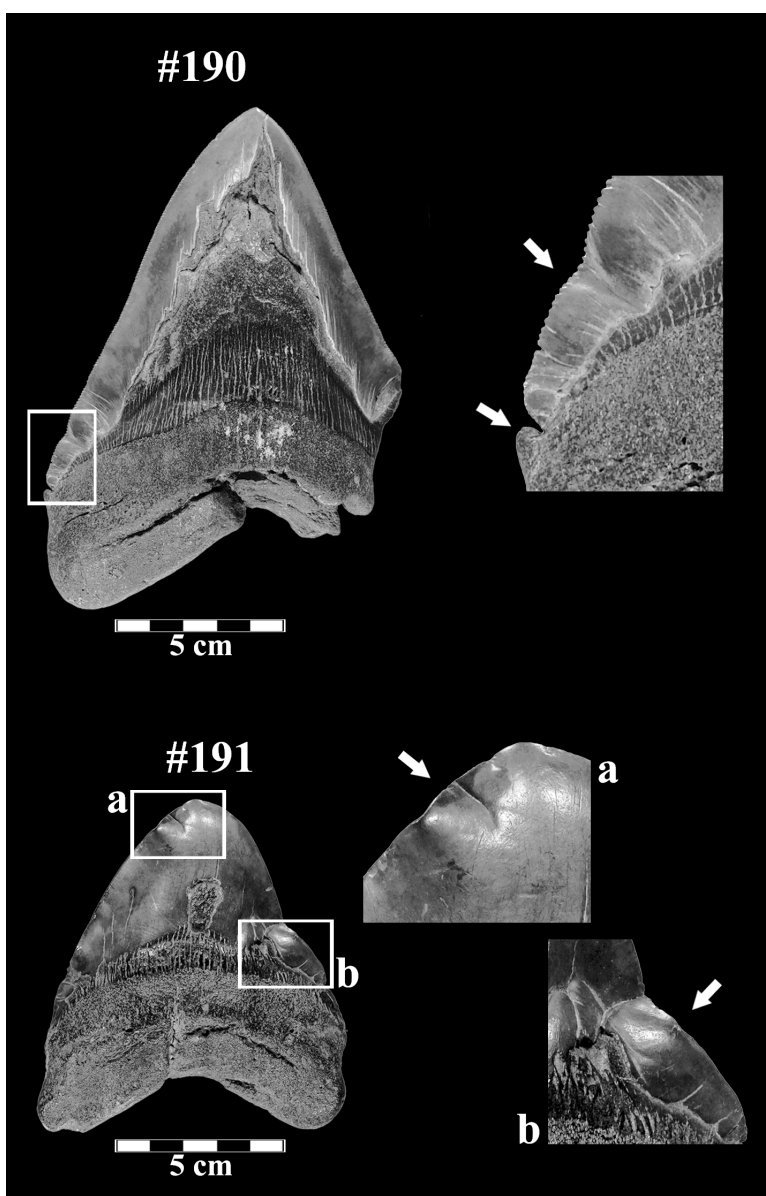

\#192
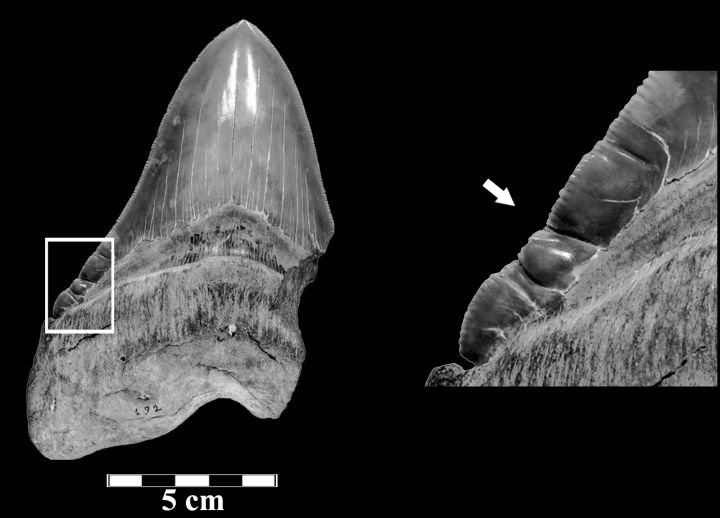

Fig. 3. Vista lingual y en detalle de los especímenes \#190, \#191 y \#192 de Carcharocles megalodon. El espécimen \#190 muestra una conformación ondulada en el margen mesial de la corona. Además, muestra una pequeña formación radicular. El espécimen \#191 muestra una superficie lingual extremadamente irregular, mostrando elevaciones y hundimientos del esmalte, que afectan también a los márgenes dentales. El espécimen \#192 muestra una conformación ondulada en el margen distal de la corona, en proximidad del cuello dental. 
de la corona en la parte inferior del margen distal. El margen mesial de la corona muestra una evidente ondulación del esmalte, en proximidad del cuello del diente. La conformación ondulada del esmalte también es parcialmente visible sobre el margen distal; sin embargo, las alteraciones tafonómicas no permiten evaluar el grado y la extensión de la deformidad. El espécimen, además, muestra una pequeña formación radicular, sobre el cuello dental, en el margen mesial (Fig. 3).

Espécimen \#191 (?; diente superior no identificado). La corona dental es anómala en su totalidad. Tanto la superficie lingual como la labial son extremadamente irregulares, mostrando elevaciones y hundimientos del esmalte, que afectan también a los márgenes dentales (Fig. 3).

Espécimen \#192 (AIII; tercer diente anterior superior). Se observan alteraciones de origen tafonómico ya sea en la corona como en la raíz. En particular, falta parte del revestimiento de esmalte lingual, en proximidad del cuello dental, así como están ausentes el lóbulo radicular y parte de la corona en la parte inferior del margen mesial. El margen distal de la corona presenta una conformación ondulada en proximidad del cuello dental (Fig. 3).

\section{DISCUSIÓN}

Los dientes examinados muestran principalmente anomalías de la corona (Tabla I). En un solo caso, el relacionado con el espécimen \#190, se observa una pequeña formación sobre la raíz que, de acuerdo con Renz, está comúnmente presente en los dientes de grandes dimensiones. No obstante, es necesario hacer distinciones dentro de las anomalías, ya que estas presentan morfologías diferentes e incluyen distintas regiones anatómicas de la corona.
Los especímenes \#118, \#190 y \#192 muestran ondulaciones de los márgenes coronales ya descritos en $C$. megalodon y diagnosticadas como deformaciones congénitas (Renz). Sin embargo, el investigador David J. Ward opina que estas deformaciones pueden ser atribuidas a la edad avanzada de los ejemplares a los que pertenecen los dientes (afirmación citada en Renz). Teniendo en cuenta que (i) la longitud media de los ejemplares adultos de $C$. megalodon es de 10,02 m (Pimiento \& Balk); (ii) la longitud promedio de los ejemplares de $C$. megalodon del Museo Universitario de Chieti es de 10,06 $\pm 2,23 \mathrm{~m}$ (Viciano et al., 2008); y (iii) el tamaño de los Lamniformes varía con la edad (Hubbell), podemos suponer que los especímenes analizados podrían haber pertenecido a ejemplares adultos y, en consecuencia, no se puede excluir que los márgenes coronales ondulados puedan ser una anomalía morfológica relacionada con la edad del animal. Por otra parte, la edad adulta de los ejemplares examinados parece ser también confirmada por la reducida visibilidad de las aserraduras de los márgenes cortantes del diente como consecuencia del desgaste físiológico.

Alteraciones macroscópicas, esta vez extendidas sobre toda la corona de los dientes, afectan a los especímenes \#188 y \#191. Renz ha publicado imágenes de casos análogos de deformación de la corona en dientes de C. megalodon procedentes de Chile, Nueva Caledonia y de Carolina del Sur (EE.UU.); sin embargo, son ausentes las descripciones de tales alteraciones.

La aposición anómala del esmalte observada sobre la superficie labial del diente \#165 representa el primer caso descrito en $C$. megalodon y, según nuestro conocimiento, en otras especies fósiles (p. ej.: C. auriculatus) o vivas (p. ej.: Carcharodon carcharias).

La hendidura del margen mesial de los dientes \#119 y \#120 podría ser el resultado de eventos traumáticos rela-

Tabla I. Dientes de Carcharocles megalodon del Museo Universitario de Chieti: clasificación y diagnóstico patológico.

\begin{tabular}{llll}
\hline Espécimen & Clasificación* & Tipo de alteración & Diagnóstico \\
\hline$\# 118$ & AII & Conformación ondulada en el margen mesial de la corona & Anomalía congénita \\
$\# 119$ & UL2 (?) & Hendidura en el margen mesial de la corona & Trauma \\
$\# 120$ & AI & Hendidura en el margen mesial de la corona & Trauma \\
$\# 165$ & Int. (?) & Aposición anómala de esmalte en la superficie labial de la corona & Anomalía congénita \\
$\# 180$ & UL6/7 & Proyección puntiforme en el marg en distal de la corona & Anomalía congénita \\
$\# 188$ & AI & Superficie labial coronal ondulada & Anomalía congénita \\
$\# 190$ & AI & Conformación ondulada en los márgenes mesial y distal de la corona. & Anomalía congénita \\
& & Pequeña formación radicular & Anomalía congénita \\
\#191 & $?$ & $\begin{array}{l}\text { Deformación generalizada de la corona, con hundimientos y } \\
\text { elevaciones del esmalte } \\
\text { Conformación ondulada en el margen distal de la corona }\end{array}$ & Anomalía congénita \\
\hline
\end{tabular}

*Para mayor información sobre el sistema de clasificación y nomenclatura dental de los especímenes analizados consultar el artículo de Viciano et al., 2018. 
cionados con la depredación. En ambos casos, la observación de las lesiones bajo el estereomicroscopio muestra una interrupción neta del margen cortante del diente, un hundimiento de la superficie del esmalte y la presencia de microfracturas.

El diagnóstico de las lesiones dentales que figuran en la Tabla I, todavía deja espacio a diferentes interpretaciones por la ausencia de un registro fósil paleopatológico de comparación, así como por los reducidos conocimientos sobre la biología y fisiología de C. megalodon (derivados casi exclusivamente de los dientes y pocos centros vertebrales hallados), y de los estudios de fisiopatología comparada (principalmente en el gran tiburón blanco Carcharodon carcharias) (Hubbell; Renz; Wroe et al., 2008).

A modo de conclusión, sería deseable incrementar el registro paleopatológico creando, en cualquier caso, una base de datos paleopatológica de referencia, basándose en el modelo de la Paleobiology Database (http://paleobiodb.org), que por el momento solo está dotada de información geológica de los yacimientos y de la fauna hallada, pero que carece de los datos métricos y paleopatológicos de los especímenes examinados.

Una colección paleopatológica extendida permitiría investigar posibles correlaciones entre las frecuencias de las diferentes alteraciones patológicas y (i) la datación y localización geográfica de los yacimientos, (ii) las dimensiones de los ejemplares de megalodonte, (iii) la tipología de los dientes afectados. Por ejemplo, la posible certificación científica de que los dientes más grandes $\mathrm{y}$, por lo tanto, pertenecientes a ejemplares de gran tamaño, presentan frecuencias más elevadas de traumas, podría sugerir una dieta de los tiburones de mayor tamaño basada en grandes mamíferos marinos (p. ej.: ballenas), diferente de aquella adoptada por los ejemplares juveniles. Además, se podría comprender si los dientes anteriores superiores son los más afectados por lesiones traumáticas precisamente porque son los primeros en impactar sobre las presas, o hay otras explicaciones plausibles.

Todavía estamos en el campo de las conjeturas, pero una muestra paleopatológica numéricamente adecuada podría proporcionar respuestas interesantes sobre la paleobiología de Carcharocles megalodon. La publicación de los datos morfológicos, métricos y paleopatológicos de la colección del Museo Universitario de Chieti (Italia) va precisamente en esta dirección.
D'ANASTASIO, R.; LÓPEZ-LÁZARO, S. \& VICIANO, J. Fossil teeth of Carcharocles megalodon: the collection of the University Museum of Chieti (Italy). Part II: Paleopathological analysis. Int. J. Morphol., 36(3):841-847, 2018.

SUMMARY: Paleopathology is the science that studies the origin and evolution of diseases. The interest in this discipline derives from the fact that the frequency and distribution of the pathologies that have affected the extinct species, correlate with their ecology and ethology. This assumption is also valid in the case of Carcharocles megalodon, the largest shark that ever existed, located at the top of the food chain and that lived in temperate seas from the middle Miocene to the Pliocene periods (15.9-2.6 My). In the present article we present the pathological alterations of the teeth of Carcharocles megalodon housed in the University Museum of Chieti (Italy). The collection consists of nine teeth of the upper arch, all of them with pathological alterations corresponding to developmental defects and crown trauma. A particular tooth (specimen \#165) presents an anomalous apposition of the enamel on the labial surface, a condition not yet described in the literature. The collection of the University Museum of Chieti enriches the existing relatively poor paleopathological record, and could improve the knowledge of the paleobiology of Carcharocles megalodon.

KEY WORDS: Megalodon; Fossil tooth; Lamniformes; Paleopathology; Congenital anomaly; Trauma.

\section{REFERENCIAS BIBLIOGRÁFICAS}

Applegate, S. P. \& Espinosa-Arrubarrena, L. The Fossil History of Carcharodon and Its Possible Ancestor, Cretolamna: A Study in Tooth Identification. In: Klimley, A. P. \& Ainley, D. G. (Eds.). Great White Sharks: The Biology of Carcharodon Carcharias. San Diego, Academic Press, 1996. pp.19-36.

Applegate, S. P. Tooth terminology and variation in sharks with special reference to the sand shark, Carcharias taurus Rafinesque. Contrib. Sci. Los Angeles Cty. Mus., 86:3-18, 1965.

Bedosti, N.; Landini, W. \& D'Anastasio, R. The increase of bony mass in a small Cyprinodontidae from the Messinian deposit of Monte Tondo (Ravenna, Italy); paleoecological implications. Atti Soc. Toscana Sci. Nat. Mem. Ser. A, 122:5-17, 2015.

Böhmer, C. \& Rössner, G. E. Dental paleopathology in fossil rhinoceroses: etiology and implications. J. Zool., 304(1):3-12, 2018.

Capasso, L. \& D'Anastasio, R. Dental caries in Gigantopithecus blaki, Late Pliocene, South China. Arch. Antropol. Etnol., 131:81-7, 2001.

Capasso, L. Principi di Storia della Patologia Umana. Roma, Società Editrice Universo, 2002.

Cilli, J. \& D'Anastasio, R. A historical case of an ameloblastoma, from southern Italy. Int. J. Paleopathol., 16:22-6, 2017.

D’Anastasio, R.; Pierfelice, L.; Di Fabrizio, A. \& Capasso, L. The cessation of tooth replacement in an aged tyrannosaur. J. Paleopathol., 14(3):1337, 2002.

D’Anastasio, R.; Wroe, S.; Tuniz, C.; Mancini, L.; Cesana, D. T.; Dreossi, D.; Ravichandiran, M.; Attard, M.; Parr, W. C.; Agur, A. \& Capasso L. Micro-biomechanics of the Kebara 2 hyoid and its implications for speech in Neanderthals. PLoS One, 8(12):e82261, 2013.

D’Anastasio, R.; Zipfel, B.; Moggi-Cecchi, J.; Stanyon, R. \& Capasso, L. Possible brucellosis in an early hominin skeleton from sterkfontein, South Africa. PLoS One, 4(7):e6439, 2009. 
Dawson, C. E. A bibliography of anomalies of fishes. Gulf Res. Rep., 1(6):308-99, 1964.

de Buffrénil, V.; Canoville, A.; D’Anastasio, R. \& Domning, D. P. Evolution of sirenian pachyosteosclerosis, a model-case for the study of bone structure in aquatic tetrapods. J. Mammal. Evol., 17(2):101-20, 2010.

De Luca, S.; Viciano, J.; Irurita, J.; López-Lázaro, S.; Cameriere, R. \& Botella, D. Mandibular fracture and dislocation in a case study from the Jewish Cemetery of Lucena (Córdoba), in South Iberian Peninsula (8th-12th AD). Int. J. Osteoarchaeol., 23(4):485-504, 2013.

Harshbarger, J. C. Neoplasm in Zoo Poikilotherms Emphasizing Cases in the Registry of Tumors In Lower Animals. In: Montali, R. J. \& Migaki, G. (Eds.). The Comparative Pathology of Zoo Animals. Washington D. C., Smithsonian Institution Press, 1980. pp.585-91.

Heupel, M. R.; Simpfendorfer, C. A. \& Bennett, M. B. Skeletal deformities in elasmobranchs from Australian waters. J. Fish Biol., 54(5):1111-5, 1999.

Hoenig, J. M. \& Walsh, A. H. Skeletal lesions and deformities in large sharks. J. Wildl. Dis., 19(1):27-33, 1983.

Honma, Y. \& Sugihara, C. A stingray, Dasyatis akajei, with aberrant pectoral fins from the Japan Sea. Jpn. J. Ichtyol., 18(4):187-9, 1971.

Hubbell, G. Using Tooth Structure to Determine the Evolutionary History of the White Shark. In: Klimley, A. P. \& Ainley, D. G. (Eds.). Great White Sharks: the Biology of Carcharodon Carcharias. San Diego, Academic Press, 1996. pp.9-18.

Leriche, M. Les poissons Néogènes de la Belgique. Mem. R. Belg. Mus. Nat. Sci., 32:365-472, 1926.

Mancini, P. L.; Casas, A. L. \& Amorim, A. F. Morphological abnormalities in a blue shark Prionace glauca (Chondrichthyes: Carcharhinidae) foetus from southern Brazil. J. Fish Biol., 69(6):1881-4, 2006.

Pimiento, C. \& Balk, M. A. Body-size trends of the extinct giant shark Carcharocles megalodon: a deep-time perspective on marine apex predators. Paleobiology, 41(3):479-90, 2015.

Purdy, R. W.; Schneider V. P.; Applegate, S. P.; McLellan, J. H.; Meyer, R. L. \& Slaughter, B. H. The Neogene Sharks, Rays, and Bony Fishes from Lee Creek Mine, Aurora, North Carolina. In: Ray, C. E. \& Bohaska, D. J. (Eds.). Geology and Paleontology of the Lee Creek Mine, North Carolina, III. Washington D. C., Smithsonian Institution Press, Smithsonian Contributions to Paleobiology, no 90, 2001. pp.71-202.

Ramírez Hernández, A. M.; Palacios Barreto, P. Y.; Gaitán Espitia, J. D.; Ramírez Tellez, J. \& Reyes, F. Morphological abnormality in the longnose stingray Dasyatis guttata (Myliobatiformes: Dasyatidae) in the Media Guajira, Colombian Caribbean. Cybium, 35(1):79-80, 2011.

Renz, M. When Things Go Wrong (Abnormalities in the Fossil Record). In: Renz, M. (Ed.). Megalodon: Hunting the Hunter. Florida, PaleoPress, 2002. pp.119-27.

Reolid, M. \& Molina, J. M. Record of Carcharocles megalodon in the Eastern Guadalquivir Basin (Upper Miocene, South Spain). Estud. Geol., 71(2):e032, 2015.

Rothschild, B. M. \& Martin, L. D. Paleopathology: Disease in Fossil Record. London, CRC Press, 1993.

Schlumberger, H. G. \& Lucke, B. H. Tumors of fishes, amphibians, and reptiles. Cancer Res., 8(12):657-753, 1948.

Uyeno, T.; Sakamoto, O. \& Sekine, H. The description of an almost complete tooth set of Carcharodon megalodon from a Middle Miocene bed in Saitama Prefecture, Japan. Saitama Mus. Nat. Hist. Bull. 7:73-85, 1989

Viciano, J.; D'Anastasio, R. \& Capasso L. Timing of maxillofacial-oral injuries in an individual of the ancient city of Herculaneum (79 AD, Naples, Italy): a case report. Dent. Traumatol., 31(3):215-27, 2015a.

Viciano, J.; D'Anastasio, R. \& Capasso, L. Odontometric sex estimation on three populations of the Iron Age from Abruzzo region (centralsouthern Italy). Arch. Oral Biol., 60(1):100-15, 2015 b.

Viciano, J.; De Luca, S.; López-Lázaro, S.; Botella, D. \& Diéguez-Ramírez, J. P. A probable case of gigantism/acromegaly in skeletal remains from the Jewish necropolis of "Ronda Sur" (Lucena, Córdoba, Spain; VIIIXII centuries CE). Anthropol. Anz., 72(1):67-87, 2015c.
Viciano, J.; López-Lázaro, S. \& D’Anastasio, R. Dientes fósiles de Carcharocles megalodon: la colección del Museo Universitario de Chieti (Italia). Parte I: Análisis morfométrico. Int. J. Morphol., 2018. En Prensa.

Viciano, J.; López-Lázaro, S.; Cesana, D. T.; D’Anastasio, R. \& Capasso, L. Multiple traumatic dental injuries: A case report in a young individual from the Samnitic necropolis of Opi Val Fondillo (VI-V century BC; Central Italy). J. Archaeol. Sci., 39(2):566-72, 2012.

Viciano, J.; Urbani, V. \& D' Anastasio, R. Congenital anatomical variant of the clavicle. Anat. Rec. (Hoboken), 300(8):1401-8, 2017.

Wroe, S.; Huber, D. R.; Lowry, M.; McHenry, C.; Moreno, K.; Clausen, P.; Ferrara, T. L.; Cunningham, E.; Dean, M. N. \& Summers, A. P.Threedimensional computer analysis of white shark jaw mechanics: how hard can a great white bite? J. Zool., 276(4):336-42, 2008.

\section{Dirección para correspondencia: \\ Ph.D. Joan Viciano}

Unidad Operativa de Antropología

Departamento de Medicina y Ciencias del Envejecimiento Universidad de "G. d'Annunzio "de Chieti-Pescara

Chieti Pescara

ITALIA

Email: joan.viciano@unich.it

Recibido : 09-02-2018

Aceptado: 17-04-2018 